DOI: $10.24850 /$ j-tyca-2018-03-04

Artículo

\title{
Evaluación estacional del riesgo por inundación en zonas agrícolas
}

\section{Seasonal flood risk assessment in agricultural areas}

Beatriz Edith Vega-Serratos ${ }^{1}$

Ramón Domínguez-Mora²

Gregorio Posada-Vanegas 3

${ }^{1}$ Instituto de Ecología, Pesquerías y Oceanografía del Golfo de México (Instituto Epomex), Universidad Autónoma de Campeche, Campus VI, San Francisco de Campeche, Campeche, México, beaevega@uacam.mx

2Instituto de Ingeniería, Universidad Nacional Autónoma de México, Ciudad de México, México, RDominguezM@iingen.unam.mx

3Instituto de Ecología, Pesquerías y Oceanografía del Golfo de México (Instituto Epomex), Universidad Autónoma de Campeche, Campus VI, San Francisco de Campeche, Campeche, México, gposadav@uacam.mx

Autor para correspondencia: Beatriz Edith Vega Serratos, beaevega@uacam.mx

\section{Resumen}

El sector agrícola en México está expuesto a diversos riesgos hidrometeorológicos, cuyos efectos negativos pueden alcanzar dimensiones de desastres, ocasionando grandes daños patrimoniales a los productores y a la economía familiar, ya sea por pérdida parcial o total de la inversión y del ingreso esperado. También pueden generar daños a la economía regional y nacional por la interrupción del ciclo productivo, reducción de los ingresos, desempleo y desabasto de alimentos, entre otros. Este trabajo presenta una metodología que permite evaluar el 
riesgo por inundación en áreas de uso agrícola. El método que se propone utiliza un modelo hidrodinámico bidimensional para obtener los parámetros del peligro natural, como son profundidad, duración y velocidad de la inundación. Se construyeron curvas de daño a partir de datos obtenidos mediante la aplicación de encuestas a personal científico, técnico y agricultores, complementadas con información bibliográfica; dichas curvas consideran las distintas etapas del ciclo vegetativo del cultivo, de manera que al relacionarlas con la estimación de la probabilidad de que una creciente ocurra en cada mes del año es posible calcular la esperanza de daño asociada con cada magnitud de la creciente. La zona de estudio corresponde a la cuenca del río Champotón, en el estado de Campeche, México, en la cual las inundaciones, al ser lentas, se caracterizan porque la duración es de mayor relevancia que la velocidad de la corriente.

Palabras clave: inundación fluvial, modelación hidrodinámica, funciones de daño en cultivos, daño agrícola, temporalidad de las inundaciones, etapas de desarrollo en cultivos, riesgo por inundación, daño anual esperado.

\section{Abstract}

The agricultural sector in Mexico is exposed to various hydrometeorological risks, the negative effects of which can reach disastrous proportions, causing significant financial loss to producers and the family economy due to the partial or total loss of the investment and the reduction in income from the sale of crops by farmers. These disasters can also damage the regional and national economy by interrupting the production cycle, reducing income, and creating unemployment and food shortages, among others. This study presents a method that evaluates the risk due to flooding in agricultural areas. The method proposed uses a two-dimensional hydrodynamic model to obtain the parameters of the natural hazard, including the depth, duration, and velocity of the flood. Damage curves were constructed from data obtained by administered surveys to scientific, technical, and farming personnel, supplemented with bibliographic information. These curves considered the various stages of the vegetative crop cycle in such a way that by relating them to the probability estimate of an extreme flow occurring each month of the year, it was possible to calculate the expected damage associated with each magnitude of the extreme flows. The study area corresponds to the Champoton River basin in the state of Campeche, Mexico, where the duration of floods, which are slow, is more important than the velocity of the water. 
Keywords: River flooding, hydrodynamic modelling, damage functions for crops, agricultural damage, flood seasonality, development stages of crops, flood risk, annually expected damage.

Recibido: 09/12/2016

Aceptado: 03/11/2017

\section{Introducción}

Los efectos devastadores de las inundaciones se reflejan tanto en el ámbito social como en el económico de un país o región. En los últimos años se ha hecho un gran esfuerzo en el mundo por identificar las causas que generan una inundación y sus características, el grado de afectación y la preparación que las poblaciones tienen ante estos fenómenos naturales. Avanzando más allá de las medidas de mitigación estructurales, se ha desarrollado el concepto de manejo integral del riesgo (van Westen, 2010). Así, las actividades asociadas con cada etapa del manejo del riesgo por inundación dependen en particular de los resultados de la evaluación económica del impacto de las inundaciones (Dutta, Herath, \& Musiake, 2003; Li, Wu, Dai, \& Xu, 2012).

En el sector agrícola, los daños económicos esperados debido a las inundaciones suelen ser considerablemente inferiores a los de las zonas urbanas para el mismo nivel de exposición (Merz, Kreibich, Schwarze, \& Thieken, 2010). Por lo anterior, aun cuando se han desarrollado varios métodos para estimar las pérdidas monetarias en el sector agrícola, la evaluación de daños en zonas rurales se ha hecho con métodos simples y aproximados (Förster, Kuhlmann, Lindenschmidt, \& Bronstert, 2008; Merz et al., 2010; Brémond, Grelot, \& Agenais, 2013). Por lo anterior, se justifica desarrollar un método que considere los elementos básicos que intervienen en el proceso productivo y permita estimar la pérdida de los cultivos debido a las inundaciones. 
Para la evaluación de los daños por inundación en el sector agrícola se han propuesto varios métodos de complejidad variable desarrollados principalmente en países europeos. Las diferencias entre las metodologías son función de la escala de análisis, tamaño del área de estudio, exactitud de análisis, cantidad de recursos requeridos, y cantidad de datos necesarios para aplicar los modelos numéricos disponibles y de los componentes del sistema agrícola considerados (Meyer \& Messner, 2005; Merz et al., 2010). De acuerdo con algunos estudios cualitativos sobre impactos de las inundaciones en la agricultura (Pivot, Josien, \& Martin, 2002; Twining et al., 2007; Posthumus et al., 2009; Chatterton, Viavattene, Morris, Penning-Rowsell, \& Tapsell, 2010; Morris \& Brewin, 2013), los elementos que pueden presentar daños directos por una inundación son los siguientes: cultivos, material vegetal perene, suelo, edificios, maquinaria, ganado, productos de origen animal y material almacenado. Los daños a la infraestructura, como carreteras y caminos, en relación con las zonas agrícolas rara vez son mencionados, pero se indican por Förster et al. (2008); Chatterton et al. (2010), y Morris y Brewin (2013). La mayoría de los trabajos que evalúan la pérdida agrícola por inundación considera principalmente los daños en los cultivos. Otros elementos como afectaciones a casas de labranza y la infraestructura agrícola, además de las pérdidas de la cosecha, se han considerado por Dutta et al. (2003). Por otro lado, Pivot et al. (2002) abordaron el tema de los efectos de las inundaciones sobre las características del suelo, el potencial de disminución de la calidad de los suelos y la pérdida de la estructura del suelo.

Para que una metodología proporcione estimaciones realistas de los daños es muy importante la selección adecuada de los parámetros del riesgo por inundación. Los más utilizados y que tienen mayor influencia en el cálculo de los daños directos son aquellos de tipo genérico y que pueden ser obtenidos a partir de modelos hidráulicos (Brémond et al., 2013). Actualmente existen varios modelos numéricos para caracterizar las inundaciones por el desbordamiento de ríos, como el HECRas (Posada, Veja, Ruiz, Echávez-Aldape, \& Martínez, 2011; Martínez, 2011); ISIS; Mike 11, y Mike Flood (Patro, Chatterjee, Mohanty, Singh, \& Raghuwanshi, 2009; Kadam \& Sen, 2012), los cuales son modelos unidimensionales (1D). Por otro lado, entre los modelos 2D más comunes se encuentran Telemac2, Mike 21, RisoSurf (Sommer et al., 2009) o TrimR2D. En todo caso, la selección del modelo numérico a utilizar dependerá de factores como la calidad de información disponible y el nivel de escala del estudio. Con respecto a los parámetros de inundación que se pueden considerar para la construcción de las funciones de daño directo en la agricultura son la estacionalidad de las inundaciones, 
profundidad del agua, duración, velocidad de la corriente, depósitos, contaminación ambiental y salinidad del agua (Brémond et al., 2013).

La importancia relativa de cada parámetro de inundación varía de una región a otra, y depende de las condiciones de inundación y las características del área de estudio. La profundidad de inundación es el parámetro más utilizado en la construcción de funciones de daño directo. En el caso de la infraestructura de vivienda, servicios y menaje, el porcentaje de daño está asociado con la altura que el agua alcanza en estas edificaciones; por otro lado, en la agricultura también se considera para evaluar los daños en el material vegetal y en algunos casos del suelo (Brémond, 2012). La estacionalidad de las inundaciones vincula diferentes coeficientes de daño para cada estación del año. Con respecto a la duración, se han considerado los días de sumersión del cultivo para evaluar el daño en el material vegetal y en algunos casos no es claro si este parámetro se refiere a los días que el suelo de la parcela tardó en secarse, lo cual depende del tipo de suelo. La velocidad es un parámetro que no es común utilizar en las funciones de daño en la agricultura, como sí lo es en la evaluación de edificaciones domésticas. En casos específicos se establecen intervalos como velocidad media, baja y alta para determinar el daño potencial de la planta (Dutta et al., 2003; Förster et al., 2008; Brémond et al., 2013). El depósito de sedimentos en las llanuras de inundación es un parámetro que puede afectar la producción, generando repercusiones en la alimentación del ganado (USACE, 1985). Por otro lado, la sal puede generar impactos específicos en los cultivos y el suelo; en el caso de la sumersión marina, el efecto provoca una alta reducción en la producción inducida por la toxicidad de la sal en el suelo (Roca et al., 2011).

Hasta ahora, ningún método ha incluido todos los parámetros mencionados en un único modelo de estimación de daños (Vozinaki, Karatzas, Sibetheros, \& Varouchakis, 2015), sin embargo se han realizado combinaciones de los parámetros más influyentes; Förster et al. (2008), y Pistrika (2010) consideraron la estacionalidad y duración; Citeau (2003) utilizó el tiempo de inmersión, velocidad y profundidad. Otras combinaciones más complejas han sido presentadas por la USACE (1985), considerando estacionalidad, profundidad del agua, duración, velocidad y depósito de sedimentos. Brémond y Grelot (2012) combinan cuatro parámetros de la inundación para construir sus funciones de daño en cultivos: estacionalidad, profundidad del agua, duración y velocidad.

En los métodos de estimación de daños, el desarrollo de las funciones de daño o curvas de daño es esencial e implica un proceso complejo (Smith 1994; Vozinaki et al., 2015). Existen dos tipos de curvas de daños: las históricas, que han sido obtenidas de bases de datos de daños de 
inundaciones pasadas como HOWAS 21, desarrollada en Alemania; y, por otro lado, las curvas de daño sintéticas, las cuales se basan en análisis hipotéticos de daños esperados bajo ciertas condiciones de inundaciones (Vozinaki et al., 2015). Diversas funciones de daño se han utilizado en el pasado en el sector agrícola, funciones que han combinado datos históricos con datos sintéticos (Merz et al., 2010); curvas derivadas de datos históricos promediados y normalizados de las publicaciones del Ministerio Japonés de la Construcción, considerando la profundidad y duración de las inundaciones (Dutta et al., 2003). Funciones empíricas basadas en datos de daños agrícolas registrados por el Ministerio Griego de Desarrollo Rural han sido utilizadas en Pistrika (2010). Brémond y Grelot (2010) construyeron funciones de daños a partir de datos obtenidos de la literatura y entrevistas. Thieken et al. (2009) han realizado encuestas en línea para obtener una base de datos de daños en el sector rural. Basados en datos empíricos, obtenidos en entrevistas a expertos, se han generado factores de impacto para diferentes tipos de cultivos (Förster et al., 2008).

El sector agrícola en México está expuesto a diversos riesgos hidrometeorológicos, cuyos efectos negativos pueden alcanzar dimensiones de catástrofe, derivando en daños patrimoniales a los productores y a la economía familiar, ya sea por pérdida parcial o total de la inversión y del ingreso esperado. De igual forma, pueden generar daños a la economía regional y nacional por la interrupción del ciclo productivo, reducción de los ingresos, desempleo y desabasto de alimentos, entre otros.

El objetivo de este trabajo es desarrollar una metodología que permita evaluar el riesgo por inundación en áreas de uso agrícola. La metodología toma como base trabajos previos adaptados a las condiciones de la zona de estudio y la información disponible. El método que se propone utiliza un modelo bidimensional para obtener los parámetros de peligro, como la profundidad, duración y velocidad de la inundación. Las funciones de daño consideran la estacionalidad de la inundación relacionada con el ciclo vegetativo del cultivo. Debido la falta de una base de datos histórica sobre daños en la agricultura se construyeron curvas de daño por medio de entrevistas aplicadas a personal científico, técnico y agricultores, completadas con información bibliográfica. La zona de estudio corresponde a la cuenca del río Champotón, en el estado de Campeche, México, en la cual las inundaciones son lentas, por lo que el parámetro de la duración es más importante que la velocidad en las áreas agrícolas. 


\section{Área de estudio}

La metodología propuesta se aplicó en la cuenca del río Champotón, ubicada en la parte central del estado de Campeche (Figura 1). Pertenece a la Región Hidrológica (RH) número 31 Yucatán Oeste; su superficie total es de $649 \mathrm{~km}^{2}$, con una elevación máxima de $120 \mathrm{msnm}$. Limita al norte con la RH número 32 Yucatán Norte, al sur con la RH número 30 GrijalvaUsumacinta, y al este y oeste con el Golfo de México.

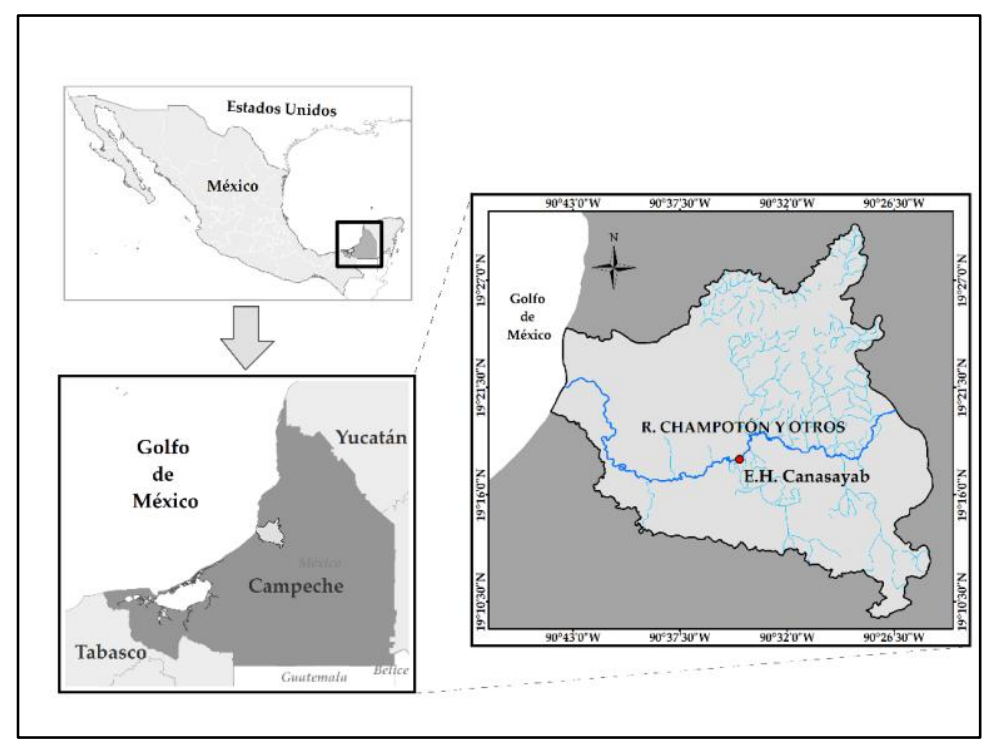

Figura 1. Zona de estudio.

La longitud del río es de unos $48 \mathrm{~km}$, con origen cerca del poblado de San Juan Carpizo hasta su desembocadura al Golfo de México (PosadaVanegas, Vega-Serratos, \& Silva-Casarín, 2013). El desarrollo principal del río es sobre una planicie de pendiente suave, con un ancho promedio de $50 \mathrm{~m} \mathrm{y}$ en la desembocadura un ancho máximo de $80 \mathrm{~m}$; su profundidad media es de $4 \mathrm{~m}$. A lo largo del río se encuentran diversos ojos de agua; corrientes intermitentes; la laguna de Nayarit de Castellot, conocida localmente como laguna de Nilúm, y la laguna de Noch; y las aguadas de Xbacab, Chuina y Hool (Ramírez, 2015). Su trayectoria general es este-oeste, y es alimentado de forma subterránea por los ríos Desempeño y Las Pozas, los cuales nacen en la Región Hidrológica 
Número 31 Yucatán Oeste, y se infiltran en dirección al río Champotón. Predominan los suelos de tipo gleysoles, con alto contenido de arcilla y baja capacidad de drenaje, lo que origina la rápida saturación e impermeabilización; hacia la costa se encuentran rendzinas líticas, que tienen una capa delgada de caliza poco profundas (Sagarpa, 2009).

\section{Metodología}

La metodología propuesta para la estimación del riesgo de pérdidas por inundación en zonas agrícolas se divide en tres secciones. La primera parte evalúa el peligro por inundación mediante la aplicación del modelo hidrodinámico bidimensional, el cual es alimentado por hidrogramas de diseño para diversos periodos de retorno, y un modelo digital topobatimétrico en el que se integra la topografía de la cuenca de análisis, la batimetría del río Champotón y la zona costera cercana a la desembocadura del río en el Golfo de México. La segunda parte del método consiste en la elaboración de las funciones de daño por inundación para los cultivos más importantes de la cuenca del río Champotón. Para esta etapa, se hizo un análisis de la producción histórica de todos los cultivos que se llevan a cabo en la zona de estudio, una investigación bibliográfica de los diferentes métodos para evaluar daños en la agricultura y las variables más importantes que consideran; además, se hicieron visitas a campo para realizar entrevistas con actores del medio (agricultores, investigadores, y funcionarios estatales y federales, cuyo trabajo se asocia con la agricultura); finalmente, tomando en cuenta dichos elementos y la información disponible, se adaptaron criterios para la construcción de las curvas de daño en cultivos; en particular, se consideró la relación entre la etapa de desarrollo del cultivo y la probabilidad de que se presenten crecientes importantes asociadas con cada etapa. La tercera etapa consiste en la construcción del modelo para evaluar el daño anual esperado por inundación en zonas agrícolas, el cual considera las primeras dos etapas, el uso de suelo y vegetación, así como los costos esperados de producción. A continuación se desarrollan los principales pasos de la metodología propuesta para evaluar el daño por inundación en zonas agrícolas (Figura 2). 


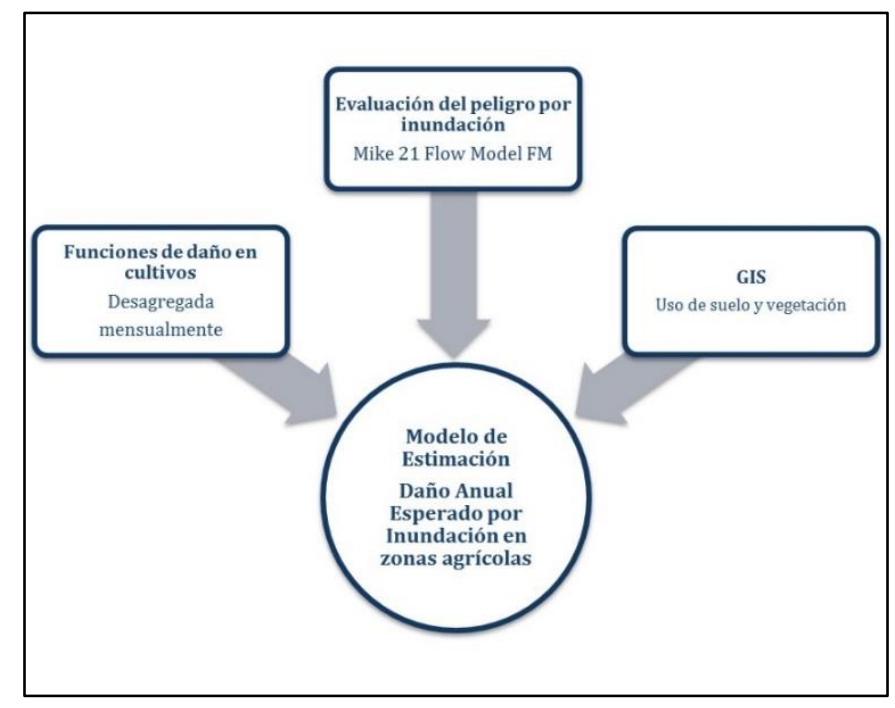

Figura 2. Diagrama de flujo de la metodología propuesta.

\section{Evaluación del peligro por inundación}

En el contexto del riesgo, el peligro se define como la probabilidad de ocurrencia de un fenómeno potencialmente dañino de cierta intensidad, durante un cierto periodo de tiempo y en un sitio dado. Es importante definir los fenómenos perturbadores mediante parámetros cuantitativos con un significado físico preciso que pueda medirse numéricamente y ser asociado mediante relaciones físicas con los efectos del fenómeno sobre los bienes expuestos (Guevara, Quaas, \& Fernández, 2006).

Para caracterizar el peligro por inundación, en este trabajo se utilizó el modelo Mike 21 Flow Model FM (DHI, Water \& Environment, 2014a), desarrollado por el Instituto Danés de Ingeniería Hidráulica, el cual contiene un sistema de modelado completo para los flujos a superficie libre en 2D aplicable a la simulación de fenómenos hidráulicos, y afines en lagos, estuarios, bahías, zonas costeras y mares (DHI, Water \& Environment, 2014b). El módulo utilizado fue el hidrodinámico (HD), para simular la variación de los niveles de agua y flujos sujetos a una variedad de condiciones de frontera en el área costera y en el río. En la Figura 3 se indica el diagrama de flujo del procedimiento aplicado. 


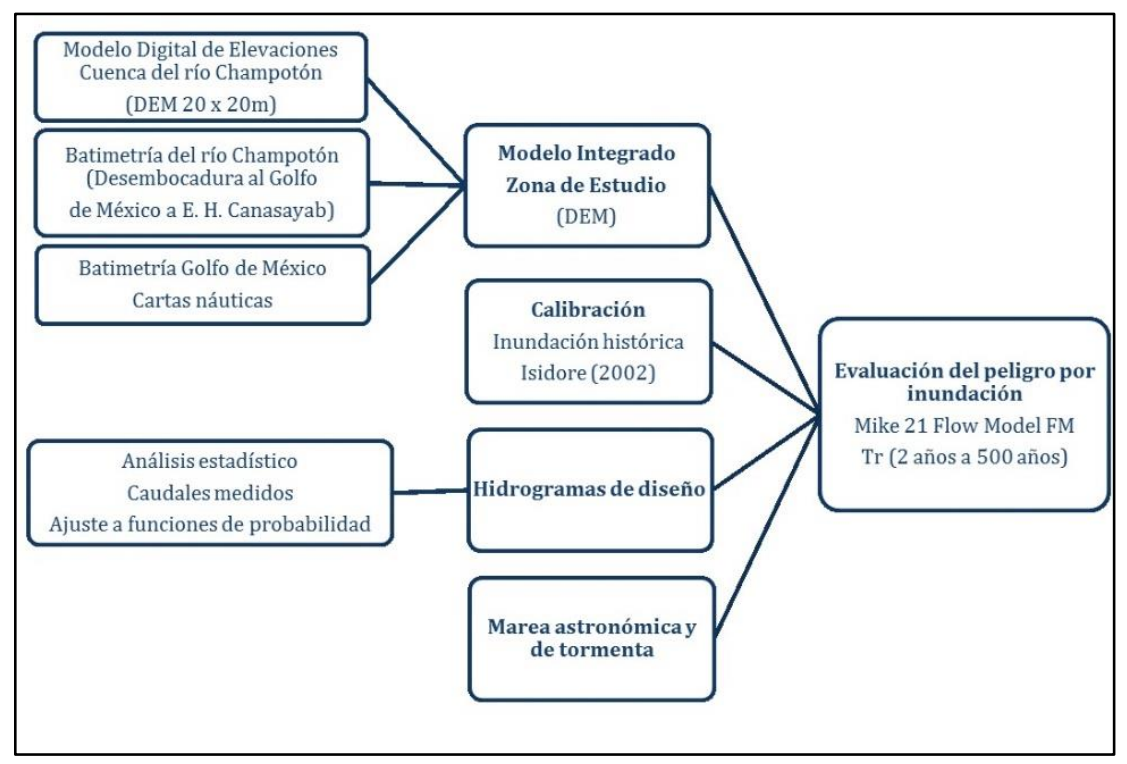

Figura 3. Diagrama de flujo de las modelaciones hidráulicas.

Se generó el modelo topobatimétrico completo ensamblando un Modelo Digital de Elevaciones (MDE, píxel de $20 \times 20 \mathrm{~m}$ ) de la cuenca del río Champotón, con la batimetría del río Champotón realizada a partir de mediciones en campo con una ecosonda y GPS de la costa del Golfo de México cercana a la desembocadura del río y la zona costera de la ciudad de Champotón (Posada-Vanegas et al., 2013); la batimetría de esta zona fue complementada con la Carta Náutica 28260 correspondiente al Golfo de México-Barra Tupilco a Isla Piedra, año 1981 de la Secretaría de Marina Armada de México (Semar) (Figura 4). El área de estudio está discretizada mediante una malla de celdas triangulares de dimensiones variables, lo que permite un mayor detalle en las zonas de interés, el río y la ciudad de Champotón, así como la desembocadura y línea de costa. El modelo se calibró con las inundaciones históricas provocadas por el huracán Isidore (2002), datos recabados en campo, marea de tormenta (PosadaVanegas et al., 2013) y el hidrograma de escurrimiento medido. 


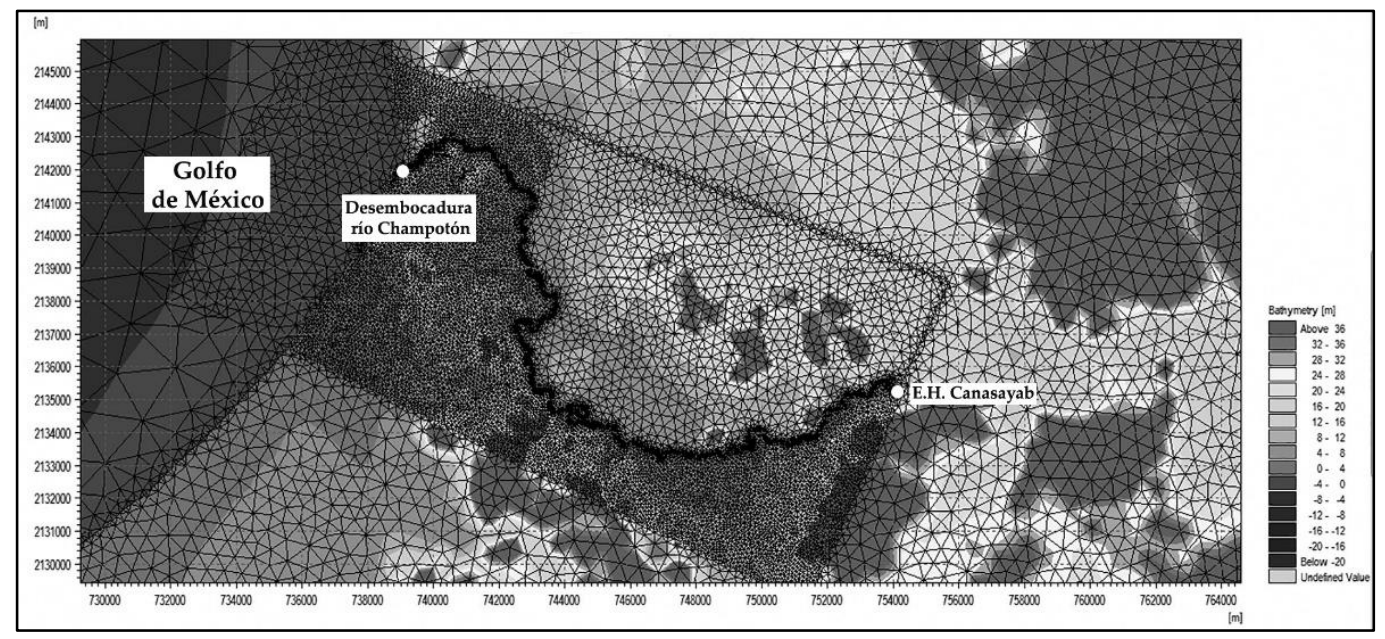

Figura 4. Malla flexible con elementos de resolución variable. Cuenca del río Champotón.

\section{Hidrogramas de diseño}

El análisis de frecuencias se utiliza como herramienta para predecir el comportamiento futuro de los caudales generados por eventos hidrometeorológicos. A partir de la información histórica y con base en procedimientos estadísticos, se calcula la magnitud del caudal asociado con un periodo de retorno ( $T r)$, el cual se define como el número de años que transcurren en promedio para que un evento de magnitud dada $x$ sea igualado o excedido por lo menos una vez en ese periodo de tiempo (Escalante-Sandoval \& Reyes-Chávez, 2002). Para la generación de los hidrogramas de diseño se aplicaron las metodologías descritas por Domínguez et al. (2008).

\section{Caudales}


Para caracterizar el peligro por inundación se utilizó el registro de caudales medidos en la estación hidrométrica Canasayab en el río Champotón durante el periodo de 1956 a 2011. Esta estación se ubica a $39 \mathrm{~km}$ de la desembocadura del río Champotón al Golfo de México. No existen ríos tributarios relevantes sobre el tramo del río entre la estación de medición y el área de análisis.

Tomando como base los registros de los gastos medios diarios de la $\mathrm{EH}$ Canasayab se hizo un análisis de frecuencias de los gastos máximos anuales. Para realizar el análisis de probabilidad se utilizó el programa $A X$ V.1.05 (Jiménez, 1992), el cual dio como resultado que la función Doble Gumbel se ajustara mejor al conjunto de datos seleccionados con el menor error estándar; después se calcularon eventos para los periodos de retorno ( $T r$ ) de 2, 5, 10, 20, 50, 100, 200 y 500 años (Figura 5).

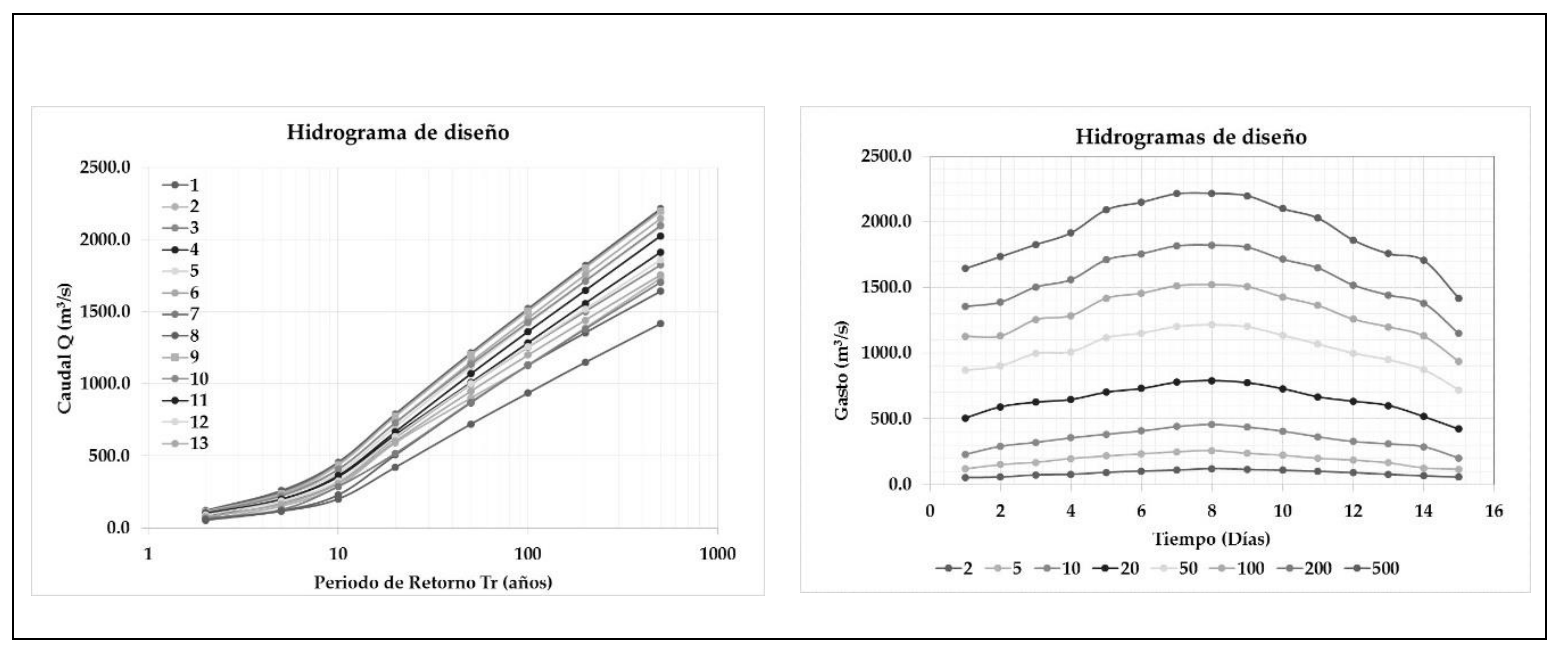

Figura 5. Análisis de frecuencia de gastos máximos anuales para diferentes duraciones.

El proceso de simulación de las inundaciones se detalla en Ramírez (2015). Como parámetros de salida de las modelaciones se utilizaron profundidad máxima del agua, duración y velocidad del flujo en cada una de las celdas de la malla flexible. 


\section{Funciones de daño por inundación en la agricultura}

El desarrollo de las funciones de daño por inundación es uno de los procedimientos más importantes en la modelación para estimar las pérdidas en la agricultura (Yu, Qin, \& Larsen, 2013). En algunos países desarrollados existen bases de datos históricos de los daños por inundaciones en los diversos sistemas afectados, lo que permite generar curvas de daño (Meyer, Scheuer, \& Haase, 2009; Yu et al., 2013; Vozinaki et al., 2015). En el caso de estudio no se cuenta con una base de datos relacionada con daños en cultivos debido a inundaciones, por lo que se adaptaron criterios y metodologías similares a las de Förster et al. (2008), Brémond et al. (2013), y Chau, Cassells y Holland (2014) para la construcción de las curvas de daño en cultivos. En la Figura 6 se muestra la secuencia propuesta para el desarrollo de las funciones.

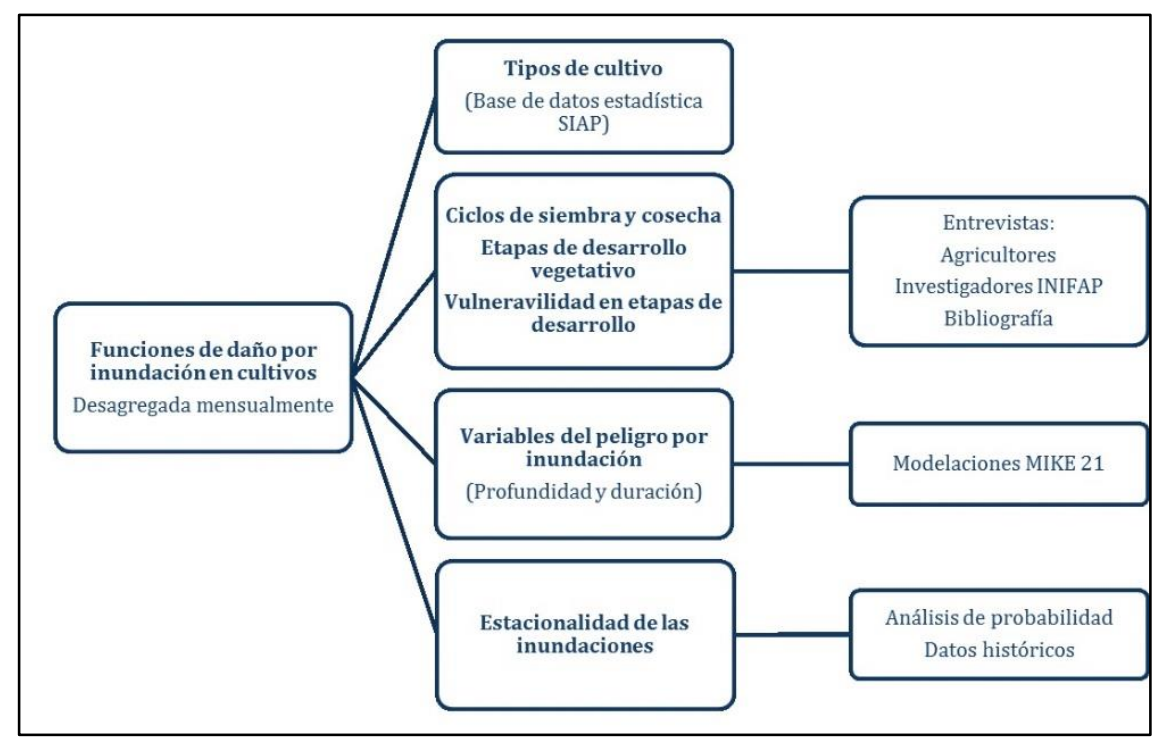

Figura 6. Diagrama de flujo para la construcción de las funciones de daño en cultivos.

\section{Parámetros del peligro por inundación que afectan a los cultivos}


La selección de los parámetros del peligro que más influyen en los daños directos en la agricultura es parte fundamental, pues de ello depende que la estimación sea real. En este trabajo, los criterios de selección de los parámetros se basan en la información recabada en campo a partir de agricultores, investigadores del Centro Experimental Edzná-INIFAP (Campeche), y en una investigación bibliográfica (Förster et al., 2008; Brémond et al., 2013; Chau et al., 2014; Vozinaki et al., 2015). De acuerdo con la información recabada y con las características de la zona de estudio, los parámetros hidráulicos que más afectan en las zonas agrícolas son la profundidad del agua, la duración de la inundación y la estacionalidad (temporalidad); con respecto a la profundidad del agua, el daño comienza con el ahogamiento de la raíz de la planta; el tiempo que se encuentra sumergida la planta, con independencia de la profundidad del agua, genera estrés hídrico; con respecto a la temporalidad, nos referimos a la etapa del crecimiento en la que están los cultivos cuando ocurre la inundación. Para la zona de estudio, debido a la baja pendiente de la zona inundable, la velocidad del flujo no es un parámetro que influya de forma significativa en el daño.

\section{Tipos de cultivos}

Como ya se ha comentado, no existe una base de datos en México asociada con inundaciones históricas que indique los daños en el caso del sector agrícola, en función de los parámetros hidráulicos que afectan principalmente a los cultivos.

Para estimar las pérdidas esperadas por inundación en las zonas agrícolas del área de estudio es necesaria la información sobre los tipos de cultivos que se llevan a cabo en las zonas cultivables; la decisión de los agricultores para seleccionar el tipo de cultivos en una región o predio depende de la relación costo-beneficio; los objetivos particulares del agricultor, y las características del suelo (Förster et al., 2008). 
En una primera etapa se consultaron fuentes oficiales de información disponibles en diferentes entidades de gobierno. La Secretaría de Agricultura, Ganadería, Desarrollo Rural, Pesca y Alimentación (Sagarpa), dentro de los servicios de consulta que maneja, ofrece el Sistema de Información Agrícola y Pecuaria, que proporciona información estadística histórica sobre los sectores productivos de México.

Para el área de análisis se extrajo la base de datos desde 1980 hasta 2013 de todos los cultivos que se practican, desagregados por ciclo primaveraverano (PV) y otoño-invierno (OI), y modalidad de riego. En la Figura 7 se observa que el maíz es el grano más representativo, seguido del sorgo, arroz palay, soya, frijol y algunas hortalizas en menor porcentaje, para la modalidad de riego de temporal. Por lo anterior, se seleccionó el cultivo de maíz para desarrollar la metodología de la construcción de las curvas de daño.

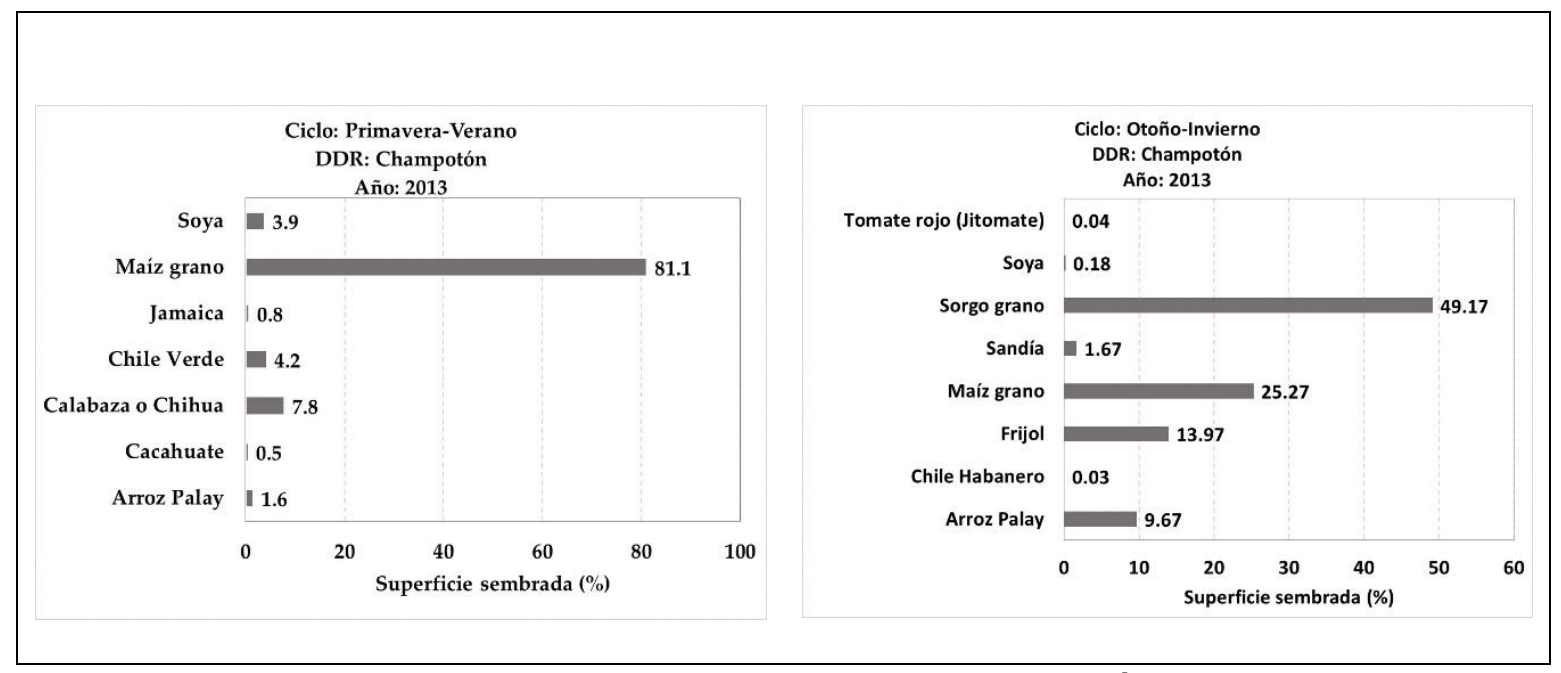

Figura 7. Superficie sembrada en el DDR Champotón, 2013. Modalidad de riego: temporal (Fuente: SIAP-Sagarpa, 2014).

\section{Etapas del crecimiento del cultivo}


El impacto del daño en los cultivos depende de la etapa del desarrollo o crecimiento en el cual se encuentre en el momento de la inundación. De acuerdo con el Centro Internacional de Mejoramiento de Maíz y Trigo (CIMMYT, abril 2016), las diferentes etapas de crecimiento del maíz se pueden dividir en dos categorías: vegetativa (V) y reproductiva (R). Además, se pueden agrupar en cuatro grandes periodos:

1. Crecimiento de las plántulas (etapas VE y V1).

2. Crecimiento vegetativo (etapas $V 2, V 3 \ldots, V n$ ).

3. Floración y fecundación (etapas VT, R0, y R1).

4. Llenado de grano y madurez (etapas R2 a R6).

En la Figura 8 se observa la escala de Ritchie y Hanway (1982), que contiene las etapas consideras en este trabajo. El número de días en cada etapa dependerá de las características particulares de la región de estudio, relacionadas con las condiciones climáticas, tipo de suelo, temperatura y variedad de la semilla, entre otros. En el Campo Experimental de Edzná-Campeche del Instituto Nacional de Investigaciones Forestales, Agrícolas y Pecuarias (INIFAP, Sagarpa) se han probado diversos materiales (semillas) en las regiones centro y norte de Campeche, incluyendo diferentes variedades, como VS-535 (nombre comercial), e híbridos como $\mathrm{H}-431$, entre otros, para determinar el rendimiento promedio de grano desde condiciones de temporal excelentes hasta adversas. 


\begin{tabular}{|c|c|c|c|c|c|c|c|c|}
\hline Fases & \multicolumn{5}{|c|}{ Fase vegetativa } & \multicolumn{3}{|c|}{ Fase reproductiva } \\
\hline $\begin{array}{l}\text { Días a inicio } \\
\text { fase después } \\
\text { de la siembra }\end{array}$ & 0 & $4-5$ & $10-15$ & $25-30$ & $30-55$ & $55-65$ & $65-90$ & $90-110$ \\
\hline Etapa & Siembra & $\begin{array}{l}\text { Germinación } \\
\text { Emergencia }\end{array}$ & Establecimiento & $\begin{array}{l}\text { Diferenciación } \\
\text { de órganos }\end{array}$ & $\begin{array}{l}\text { Alargamiento } \\
\text { entre nudos }\end{array}$ & $\begin{array}{c}\text { Floración, } \\
\text { polinización } \\
\text { y fecundación }\end{array}$ & Llenado grano & $\begin{array}{l}\text { Madurez } \\
\text { fisiológica }\end{array}$ \\
\hline Descripción & $\begin{array}{l}\text { La semilla } \\
\text { posee } 5 \text { hojas } \\
\text { preformadas } \\
\text { y una raíz } \\
\text { principal } \\
\text { llamada } \\
\text { radícula }\end{array}$ & $\begin{array}{c}\text { Germinación: } \\
\text { la radicula } \\
\text { atraviesa el } \\
\text { grano. } \\
\text { Emergencia: } \\
\text { el colióptilo } \\
\text { emerge del } \\
\text { suelo }\end{array}$ & $\begin{array}{l}\text { En el estado de } \\
\text { 2-3 hojas, la } \\
\text { planta } \\
\text { comienza a } \\
\text { fotosintetizar y } \\
\text { ya no depende } \\
\text { de las reservas } \\
\text { del grano. }\end{array}$ & $\begin{array}{l}\text { Cuando la planta } \\
\text { tiene de 6-8 hojas } \\
\text { desarrolladas, la } \\
\text { yema apical se } \\
\text { transforma en } \\
\text { inflorescencia } \\
\text { masculina (panícula), } \\
\text { las yemas axilares se } \\
\text { transforman en } \\
\text { inflorescencia } \\
\text { femenina (espiga } \\
\text { engrosada) }\end{array}$ & $\begin{array}{l}\text { Los entrenudos } \\
\text { del tallo se } \\
\text { alargan } \\
\text { rápidamente, } \\
\text { se desarrolla la } \\
\text { panícula y la } \\
\text { espiga. }\end{array}$ & $\begin{array}{c}\text { Masculina: la } \\
\text { panícula se } \\
\text { hace visible y } \\
\text { las anteras } \\
\text { comienzan a } \\
\text { liberar polen. } \\
\text { Femenina: } \\
\text { aparición de } \\
\text { estigmas, } \\
\text { ocurrela } \\
\text { polinización y } \\
\text { fecundación } \\
\text { de óvulos. }\end{array}$ & $\begin{array}{l}\text { Los granos } \\
\text { acumulan } \\
\text { almidón, } \\
\text { proteínas y } \\
\text { lípidos pasando } \\
\text { por un estado } \\
\text { lechoso, pastoso } \\
\text { yduro de } \\
\text { acuerdo a su } \\
\text { contenido de } \\
\text { agua y materia } \\
\text { seca. }\end{array}$ & $\begin{array}{l}\text { Los granos } \\
\text { finalizaron su } \\
\text { llenado. Un } \\
\text { punto negro } \\
\text { aparece en su } \\
\text { base en el } \\
\text { sitio de unión } \\
\text { con la tusa. La } \\
\text { humedad del } \\
\text { grano es } \\
\text { generalmente } \\
\text { inferior al } 40 \%\end{array}$ \\
\hline
\end{tabular}

Figura 8. Estados fenológico de la planta de maíz. Adaptado de Ritchie y Hanway (1982).

Fechas de siembra y cosecha

Para ambos ciclos agrícolas, primavera-verano y otoño-invierno, las superficies sembradas con los principales granos son bajo condiciones de temporal. La siembra se realiza en el intervalo del $1^{\circ}$ de junio al 31 de julio. Cuando se utiliza el sistema de labranza tradicional del suelo se comienza a sembrar hasta que el temporal está bien establecido, siendo el periodo óptimo del 15 de junio al 15 de julio. Con el sistema de cero labranza, esta labor puede realizarse desde las primeras lluvias, pues con el "mantillo" de residuos vegetales que queda sobre el suelo, la humedad se conserva por más tiempo (Campo Experimental Edzná Campeche, INIFAP, marzo 2015). Para el ciclo agrícola P-V del año 2015, en la Figura 9 se muestra el calendario de siembra, cosecha y comercialización para la producción de los cultivos de maíz, sorgo, arroz y soya. 


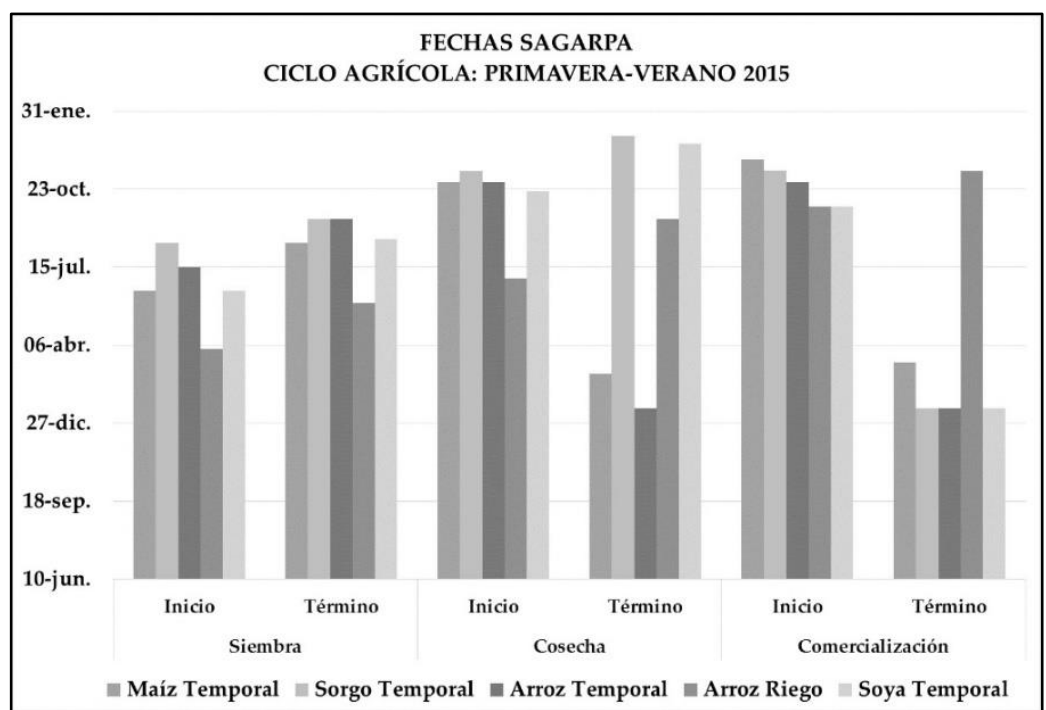

Figura 9. Fechas de producción agrícola, estado de Campeche. Ciclo PV, 2015. Fuente: Sagarpa, oficina local Campeche.

\section{Impacto del daño}

El impacto del daño depende principalmente de la etapa de desarrollo de cada cultivo en la cual se presenta la inundación. Los valores del impacto van de 0 a $100 \%$. A partir del calendario de siembra, cosecha y comercialización (Sagarpa, 2015) se desagregaron de manera mensual las etapas de desarrollo del cultivo.

En la construcción de las funciones de daño se consideraron los parámetros hidráulicos de profundidad, duración y temporalidad de la inundación. Para las diferentes profundidades de inundación se establecieron cuatro rangos de duración de la inundación. Tomando como base los estudios de Vozinaki et al. (2015), Brémond et al. (2013), y Förster et al. (2008) se construyó una función de daño para cada rango (ver Tabla 1). 
Tabla 1. Formato de tabla de encuesta para determinar el daño por inundación en cultivos.

\begin{tabular}{|c|c|c|c|c|}
\hline \multicolumn{5}{|c|}{ \% de impacto del daño en cultivo por inundación } \\
\hline \multirow{2}{*}{ Mes } & \multicolumn{4}{|c|}{ Duración (días) } \\
\hline & 1 a 3 & 4 a 7 & 8 a 11 & $>11$ \\
\hline Enero & $\ldots$ & $\ldots$ & $\ldots$ & $\ldots$ \\
\hline Febrero & $\ldots$ & $\ldots$ & $\ldots$ & $\ldots$ \\
\hline Marzo & $\ldots$ & $\ldots$ & $\ldots$ & $\ldots$ \\
\hline Abril & $\ldots$ & $\ldots$ & $\ldots$ & $\ldots$ \\
\hline Mayo & $\ldots$ & $\ldots$ & $\ldots$ & $\ldots$ \\
\hline Junio & $\ldots$ & $\ldots$ & $\ldots$ & $\cdots$ \\
\hline$\ldots$ & ... & $\cdots$ & ... & $\cdots$ \\
\hline Diciembre & $\ldots$ & $\ldots$ & $\ldots$ & $\ldots$ \\
\hline
\end{tabular}

Durante 2012 y 2013 se efectuaron visitas a campo, que permitieron recabar información de agricultores dedicados al cultivo de maíz, caña de azúcar y sorgo, principalmente; por otro lado, se entrevistó a investigadores del Instituto Nacional de Investigaciones Forestales, Agrícolas y Pecuarias (INIFAP), del Campo Experimental Edzná, que han realizado estudios de los diferentes cultivos de la región en el estado de Campeche, y proporcionaron sus opiniones y experiencias sobre los efectos que una inundación produce en cultivos como soya y maíz. A través de los paquetes tecnológicos para sembrar maíz de temporal en el estado de Campeche, 2015 (Sagarpa-INIFAP, Campo Experimental Edzná), se complementó la información.

Las consideraciones generales para la zona de estudio son:

- Los eventos considerados son de inundaciones lentas, es decir, la velocidad del flujo es pequeña.

- $\quad$ El drenaje del agua, producto de las inundaciones, es lento.

- Se considera un solo tipo de cultivo en cada parcela o área de cultivo. 
- $\quad$ Se evalúa el daño directo en los cultivos.

- En las zonas de estudio, la pendiente del terreno es baja, del orden de $0.1 \%$.

- El suelo en el área de estudio es de tipo gleysol mólico y gleysol éutrico, es decir, con baja permeabilidad.

- Los rangos de inundación se consideran desde $h>0 \mathrm{~m}$, tomando en cuenta que el estrés hídrico de la planta ha comenzado con la elevación del nivel freático.

Además, en el calendario que maneja la Sagarpa en el año 2015, para el ciclo P-V, la época de siembra se establece en el intervalo de junio a julio; por lo tanto, para este trabajo se considera que el proceso de producción se inicia desde mayo con la preparación del terreno; después de la cosecha del ciclo P-V se inician los preparativos para el ciclo O-I.

\section{Uso de suelo}

Para delimitar las zonas agrícolas en el área de estudio se utilizó el conjunto de datos vectoriales de uso de suelo y vegetación del Instituto Nacional de Estadística y Geografía (INEGI), escala 1:250,000, serie V (2011-2012), actualizada con imágenes de satélite Landsat 2011. De esta información se obtuvieron los polígonos dedicados a la agricultura y del tipo de cobertura vegetal, respectivamente (Figura 10). 


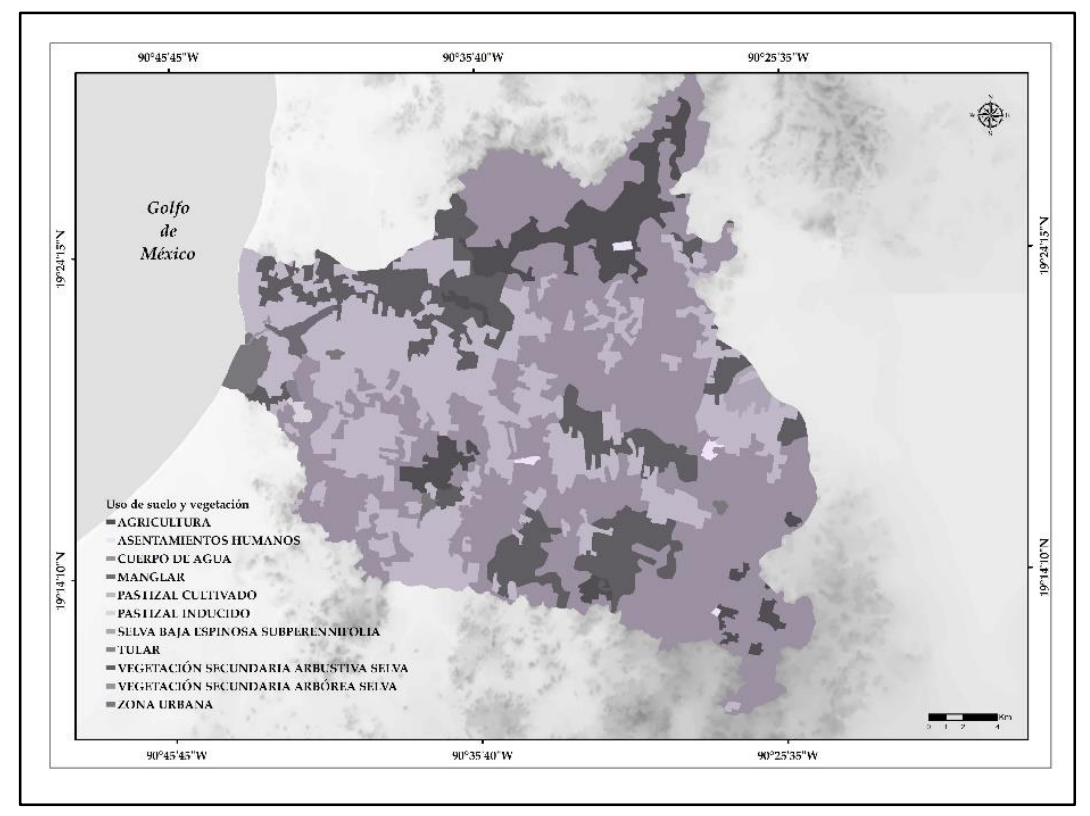

Figura 10. Uso de suelo y vegetación en la cuenca del río Champotón.

\section{Estimación del daño}

Existen diversos modelos que estiman el daño por inundación en la agricultura, cuyas diferencias radican en el número de variables que consideran $y$, al final, en la existencia o disponibilidad de información medida para que se lleve a cabo su aplicación. En este trabajo se propone evaluar el daño agrícola ocasionado por una inundación, desagregando mensualmente el impacto del daño y los costos de producción del cultivo en función de las etapas de su desarrollo mediante el siguiente modelo:

1. Se estima la probabilidad de que se presente un gasto $Q$ asociado con un periodo de retorno ( $T r$ ) en cada uno de los meses del año; para hacer la estimación, se consideró que dicha probabilidad fuera proporcional a los gastos medios mensuales registrados en la estación Canasayab. El dato que se genera es el valor de una probabilidad $P(i)$ para cada mes $i$.

2. Se obtiene el porcentaje de daño del cultivo (valor de $0-1$ ) para cada mes ante una inundación provocada por un gasto asociado con un $\mathrm{Tr}$, 
$\left(Q_{T r}\right)$. Este dato se extrae de la función del impacto del daño propuesta para las diferentes duraciones de inundación. Se proponen cuatro intervalos de duración $d$ de la inundación: $1-3 d, 4-7 d, 8-11 d$ y $>11 d$. A los datos que se generan se les denomina $\% D(i, d)$.

3. Se determina el valor del costo por hectárea ( $\$ /$ ha) que tiene el cultivo en el mes de acuerdo con la etapa de desarrollo en el proceso de producción. A los valores obtenidos se les denomina $C P H C(i)$.

4. Para cada inundación asociada con un $\operatorname{Tr}$ y cada intervalo de duración se estima un daño anual, $D C_{d}$, mediante la suma ponderada de los daños correspondientes a los 12 meses del año:

$$
D C_{d}=\sum_{i=1}^{n} P_{i} \cdot \% D_{i, d} \cdot C P H C_{i}
$$

5. Se determina el área inundada por un gasto $Q$ asociado con un $\operatorname{Tr}$ para los diferentes intervalos de duración. Se denomina a esta variable $A C I(j)$ en ha, donde $j$ varía en función del número de $\operatorname{Tr}$ analizados.

6. Se realiza el cálculo del daño, denominado $D$, para cada intervalo de duración:

$$
D\left(Q_{T r}\right)=D C_{d} \cdot A C I_{j}
$$

7. Para cada intervalo de duración se construyen las curvas de riesgo; cada punto de la curva tiene coordenadas $\left(D\left(Q_{T r}\right), T r\right)$.

8. Se obtiene el daño anual esperado como el área bajo la curva de riesgo para cada intervalo de duración (Meyer, Priest, \& Kuhlicke, 2012):

$$
D A E=\sum_{j=1}^{m} \frac{D\left(P_{T r j-1}\right)+D\left(P_{T r j}\right)}{2} * \Delta P_{j}
$$

Donde $\Delta P_{j}$ es el incremento de la probabilidad de excedencia entre periodos de retorno $(\mathrm{Tr})$.

9. El daño total promedio se obtiene mediante la suma de cada intervalo de duración. Se denomina DTP.

10. Se construye la curva de riesgo con el daño total promedio para cada $\operatorname{Tr}(D T P, T r)$.

11. El área bajo la curva de riesgo da como resultado el daño anual esperado promedio, DAEP.

\section{Resultados y discusión}




\section{Modelación hidrodinámica}

Las modelaciones realizadas con el programa hidrodinámico permitieron caracterizar el peligro por el desbordamiento del río Champotón, así como las llanuras de inundación. Se obtuvieron los valores de los tirantes máximos, velocidades y duración de las inundaciones para cada celda de la malla flexible. Para la calibración del modelo se utilizó el hidrograma medido del huracán Isidore (2002), contrastando los resultados modelados de alturas de inundación con los obtenidos en campo mediante entrevistas realizadas a los residentes de las colonias y poblaciones rurales afectadas. Los sitios donde se levantaron las encuestas de daños se muestran en la Figura 11.

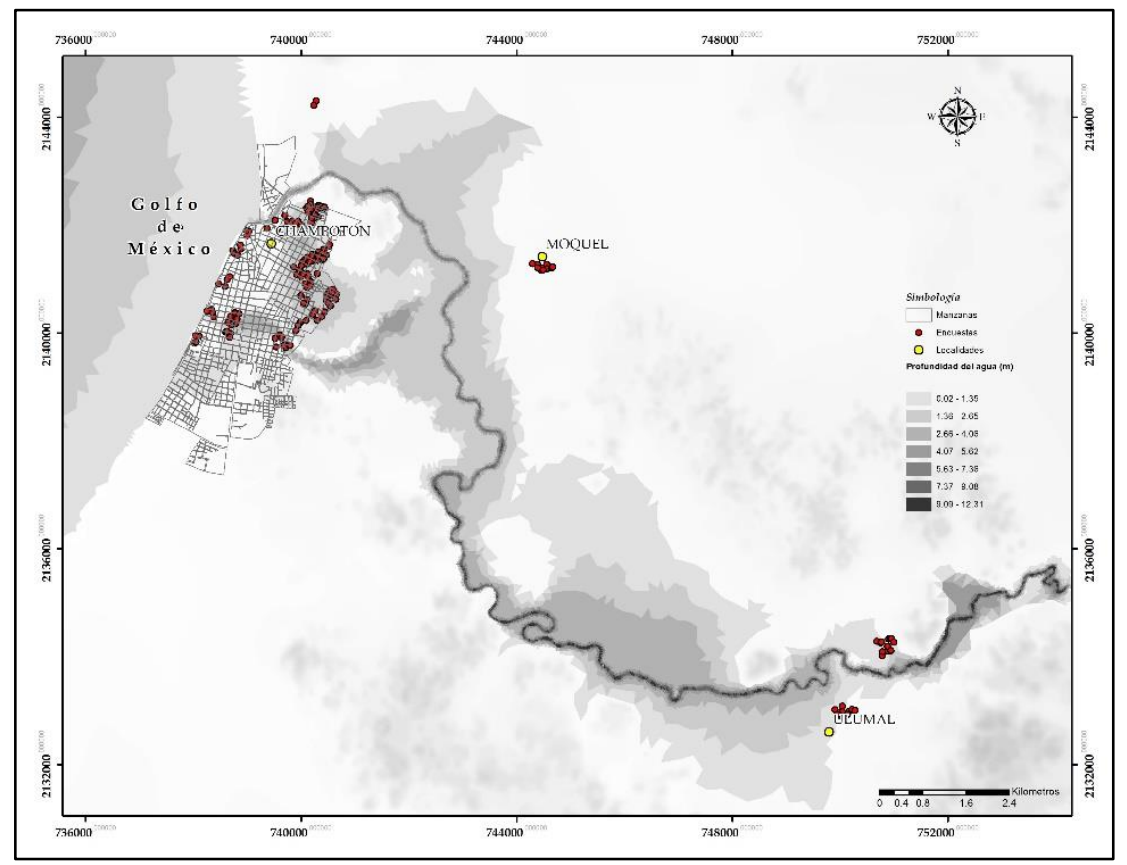

Figura 11. Resultados de la modelación hidráulica, profundidades máximas del agua para Isidore, 2002. 


\section{Temporalidad de la inundación}

Considerando que en la obtención del daño agrícola el costo de producción y el impacto del daño en los cultivos se han desagregado sobre una base mensual, de forma similar se realizó el análisis histórico de los gastos de la EH Canasayab (1956-2011). La Figura 12 muestra la distribución de escurrimientos mensuales promedio de todo el registro. De acuerdo con Agraz-Hernández et al. (2015) se identifican tres épocas climáticas en el estado de Campeche: secas, lluvias y nortes. Se observa que durante la época de secas (febrero a abril), la magnitud de los caudales pico es relativamente pequeña, pero el impacto del daño es mayor. Por otro lado, la época definida como de lluvias se encuentra bien establecida para los meses de mayo a octubre, y de acuerdo con el análisis de frecuencias, los gastos máximos anuales se concentran en los meses de septiembre y octubre, principalmente. Es importante comentar que en la época definida como de "nortes", la humedad es acompañada con fuertes vientos fríos que bajan del noreste, los cuales se presentan dentro de los meses de noviembre a enero, que si bien el orden de magnitud en los gastos pico es pequeño comparada con los de la época de lluvias, por existir una humedad antecedente del suelo, la vulnerabilidad en las zonas agrícolas es considerable.

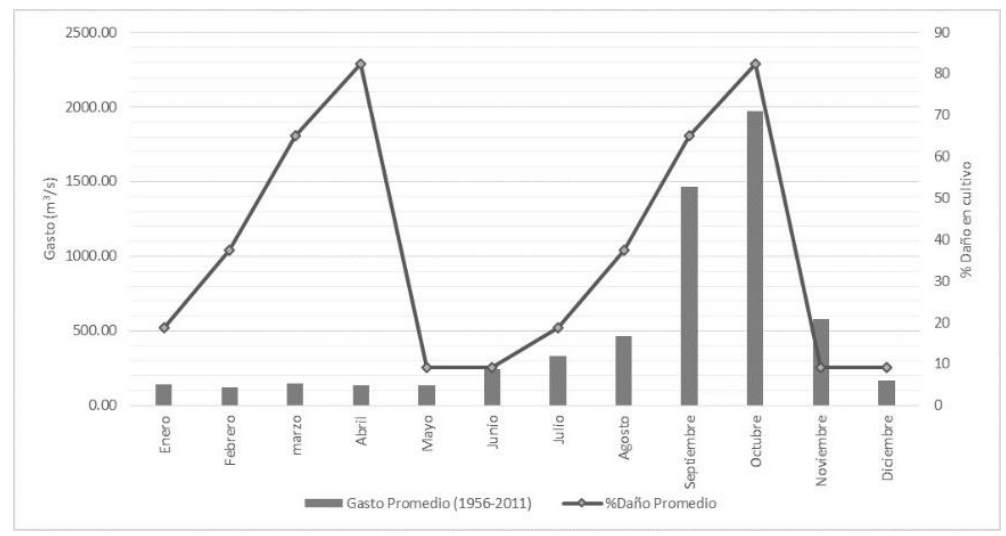

Figura 12. Caudales de la EH Canasayab (1956-2011). 
Se consideró importante desarrollar una desagregación mensual en la probabilidad de ocurrencia de una inundación por la fuerte dependencia de las pérdidas en la agricultura según el mes de ocurrencia de las inundaciones (Förster et al., 2008). En la Tabla 2 se muestran los valores de los escurrimientos medios mensuales y sus valores relativos al total anual; las inundaciones extremas asociadas con un caudal pico máximo se presentan en los meses de septiembre y octubre; a su vez, en estos meses, de acuerdo con la función promedio del daño en el maíz (Figura 12), se tiene la etapa más vulnerable para el ciclo P-V del cultivo, justo antes y durante la cosecha.

Tabla 2. Escurrimientos históricos mensuales. Estación Canasayab.

\begin{tabular}{|c|c|c|c|c|c|c|c|c|c|c|c|c|}
\hline Mes & Ene. & Feb. & Marzo & Abril & Mayo & Junio & Julio & Ago. & Sep. & Oct. & Nov. & Dic. \\
\hline $\begin{array}{c}\text { Gasto } \\
\text { promedio } \\
\text { mensual }\end{array}$ & 142.98 & 121.82 & 145.78 & 137.08 & 133.75 & 242.14 & 331.17 & 466.43 & 1465.52 & 1971.99 & 579.30 & 164.88 \\
\hline$P(i)$ & 0.024 & 0.021 & 0.025 & 0.023 & 0.023 & 0.041 & 0.056 & 0.079 & 0.248 & 0.334 & 0.098 & 0.028 \\
\hline
\end{tabular}

Funciones de daño y costos de producción

Siendo el maíz uno de los principales cultivos de la zona de estudio, el caso de análisis se aplicó sólo para aquellos predios donde se identificó este tipo de cultivo. El impacto del daño se dividió en cuatro categorías, en función de la duración de la inundación en las diferentes etapas de desarrollo del cultivo. De acuerdo con los resultados de las entrevistas, en los meses de mayo y junio, el impacto del daño es pequeño para los rangos de duración de 1 a 3 días y de 4 a 7 días de inundación, pues las afectaciones sólo implican un retraso en la preparación del terreno y en la fecha de siembra. Por otro lado, los porcentajes de daño se incrementan de modo considerable para los distintos rangos de duración entre los meses de julio a agosto, que coinciden con un periodo crítico de desarrollo del cultivo, que va de los 45 a 75 días de edad de la planta, pues es donde el cultivo se desarrolla en altura y se establece la floración; después, entre septiembre y octubre, se presenta el llenado de grano y comienza la 
cosecha, dependiendo de las condiciones de humedad del grano; en esta etapa, el impacto del daño que se maneja es de pérdida total (Figura 13).

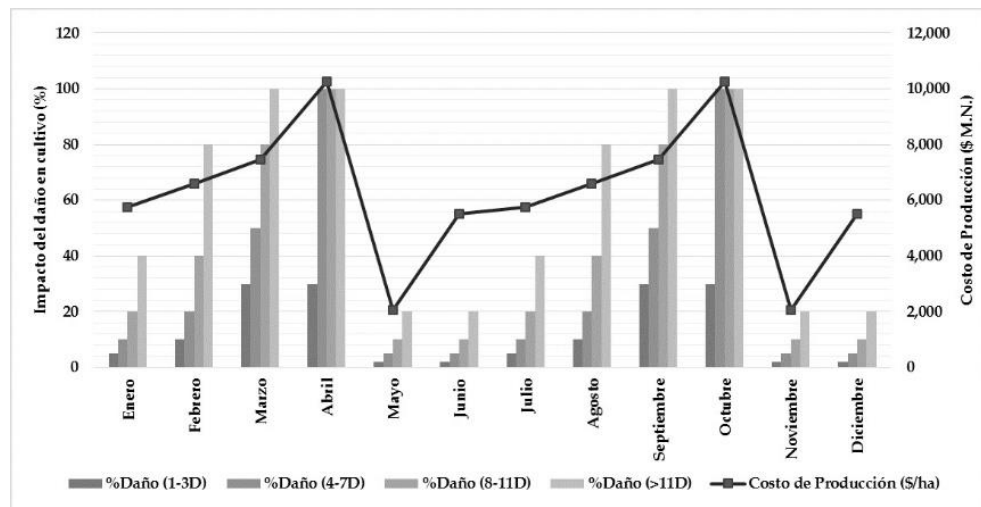

Figura 13. Impacto del daño y costos de producción en cultivo de maíz. Fuente de los costos: campo experimental Edzná-INIFAP, 2015.

Se consideran los costos directos del proceso de producción desagregados mensualmente (Figura 13). Conforme se desarrolla el cultivo se van implementando diversas actividades, acumulando el costo que implica cada una de éstas. Destacan las siguientes acciones: preparación del suelo, costos de semilla, fertilización, combate de malezas, combate de plagas, cosecha.

\section{Estimación de daño}

La evaluación del daño por inundación en zonas agrícolas se obtiene con las ecuaciones (1) a (3), mediante la interacción de las mallas de resultados de las modelaciones del peligro, uso de suelo y vegetación, las funciones de daño por tipo de cultivo y los costos de los procesos de producción.

Se inicia el proceso de cálculo con base en la malla del MDE. En cada simulación hidráulica con cierto periodo de retorno $(T r)$ se obtiene una 
malla de resultados; cada celda tiene información del tirante máximo de inundación, su duración y velocidad máxima; posteriormente se identifica para cada celda el uso de suelo, como se muestra en la Figura 10. Se aplican en cada celda las funciones de daño, de acuerdo con el intervalo de duración, y los costos de producción obtenidos del paquete tecnológico para sembrar maíz de temporal en el estado de Campeche (Figura 13). A continuación se aplica la metodología propuesta para un escenario de inundación, con un periodo de retorno (Tr) de 10 años.

La Tabla 3 indica, para cada escenario analizado en la simulación hidráulica, la magnitud de las áreas destinadas al cultivo y desagregadas por el rango de duración de la inundación.

Tabla 3. Áreas de cultivo inundadas.

\begin{tabular}{|c|c|c|c|c|}
\hline \multirow{2}{*}{ Tr } & \multicolumn{4}{|c|}{ Áreas (ha) } \\
\cline { 2 - 5 } (años) & \multicolumn{2}{|c|}{ Duración de la inundación (días) } \\
\cline { 2 - 5 } & $\mathbf{1 - 3}$ & $\mathbf{4 - 7}$ & $\mathbf{8 - 1 1}$ & $\begin{array}{c}\text { Mayor a } \\
11\end{array}$ \\
\hline 2 & 0.1488 & 1.96847 & 2.46554 & 6.4838 \\
\hline 5 & 0.25843 & 2.0113 & 4.08826 & 22.42063 \\
\hline 10 & 0.51453 & 3.15503 & 4.20933 & 37.32747 \\
\hline 20 & 0.65314 & 3.67466 & 5.6057 & 48.87276 \\
\hline 50 & 0.9211 & 9.01778 & 14.08794 & 52.27633 \\
\hline 100 & 1.87926 & 16.56971 & 27.21081 & 79.03159 \\
\hline 200 & 11.5739 & 21.13848 & 39.96751 & 81.53425 \\
\hline 500 & 21.4516 & 48.66245 & 52.79741 & 97.70343 \\
\hline
\end{tabular}

En una primera etapa de la evaluación se obtiene el daño $D\left(Q_{T r}\right)$, considerando la probabilidad $P(i)$ de que se presente una inundación en los diferentes meses del año; el porcentaje del impacto del daño, $\% D(i$, d) de una inundación en el mes $i$ con una cierta duración; y el costo de producción del cultivo $C P H C(i)$ en el mes $i$ de desarrollo. Es decir, desagregados mensualmente y obtenidos para cada área cultivada 
inundada $A C I(j)$, en cada escenario, $Q(T r)$, e intervalo de duración, $d(i)$ (Tabla 4).

Tabla 4. Evaluación del daño en áreas de cultivo de maíz. Escenario Tr: 10 años.

\begin{tabular}{|c|c|c|c|c|c|c|c|c|c|c|c|c|c|}
\hline \multicolumn{2}{|c|}{ Mes } & Enero & Febrero & Marzo & Abril & Mayo & Junio & Julio & Agosto & Sep. & Oct. & Nov. & Dic. \\
\hline \multicolumn{2}{|c|}{$\begin{array}{c}\text { Pmes, la } \\
\text { probabilidad que se } \\
\text { presente en el mes } \\
i\end{array}$} & 0.024 & 0.021 & 0.025 & 0.023 & 0.023 & 0.041 & 0.056 & 0.079 & 0.248 & 0.334 & 0.098 & 0.028 \\
\hline \multicolumn{2}{|c|}{$\begin{array}{c}\text { \% impacto daño } \\
(1-3 d)\end{array}$} & 5 & 10 & 30 & 30 & 2 & 2 & 5 & 10 & 30 & 30 & 2 & 2 \\
\hline \multicolumn{2}{|c|}{$\begin{array}{c}\text { \% impacto daño } \\
(4-7 d)\end{array}$} & 10 & 20 & 50 & 100 & 5 & 5 & 10 & 20 & 50 & 100 & 5 & 5 \\
\hline \multicolumn{2}{|c|}{$\begin{array}{c}\text { \% impacto daño } \\
(8-11 d)\end{array}$} & 20 & 40 & 80 & 100 & 10 & 10 & 20 & 40 & 80 & 100 & 10 & 10 \\
\hline \multicolumn{2}{|c|}{$\begin{array}{c}\% \text { impacto daño (> } \\
11 d)\end{array}$} & 40 & 80 & 100 & 100 & 20 & 20 & 40 & 80 & 100 & 100 & 20 & 20 \\
\hline \multicolumn{2}{|c|}{$\begin{array}{c}\text { Costo de } \\
\text { producción ( } \$ / \mathrm{ha})\end{array}$} & $5,740.00$ & $6,596.00$ & $7,452.00$ & $10,252.00$ & $2,050.00$ & $5,500.00$ & $5,740.00$ & $6,596.00$ & $7,452.00$ & $10,252.00$ & $2,050.00$ & $5,500.00$ \\
\hline $\begin{array}{l}\text { Duración } \\
\text { (días) }\end{array}$ & ACI (ha) & \multicolumn{12}{|c|}{ Daño(\$) } \\
\hline $1-3 d$ & 0.5145 & 3.58 & 7.00 & 28.41 & 36.75 & 0.48 & 2.32 & 8.28 & 26.82 & 285.59 & 528.67 & 2.07 & 1.58 \\
\hline $4-7 d$ & 3.1550 & 43.87 & 85.90 & 290.32 & 751.13 & 7.33 & 35.59 & 101.60 & 328.88 & $2,918.62$ & $10,805.78$ & 31.74 & 24.24 \\
\hline 8-11d & 4.2093 & 117.05 & 229.20 & 619.74 & $1,002.13$ & 19.55 & 94.97 & 271.11 & 877.56 & $6,230.27$ & $14,416.70$ & 84.69 & 64.67 \\
\hline$>11 d$ & 37.3275 & $2,075.99$ & $4,065.07$ & $6,869.63$ & $8,886.71$ & 346.77 & $1,684.30$ & $4,808.28$ & $15,563.95$ & $69,060.98$ & $127,844.28$ & $1,501.95$ & $1,146.92$ \\
\hline
\end{tabular}

Para cada intervalo de duración se realiza la suma del daño desagregado mensualmente, obteniendo los valores totales para el escenario de $\operatorname{Tr}=$ 10 años, así como también el daño total promedio DTP de todos los intervalos de duración (Tabla 5).

Tabla 5. Daño por intervalo de duración de la inundación en cultivo de maíz. Escenario $\operatorname{Tr}=10$ años. 


\begin{tabular}{|c|c|c|c|c|c|}
\hline $\begin{array}{c}\text { Probabilidad } \\
\text { de excedencia } \\
(\boldsymbol{P}=\mathbf{1} / \mathbf{T r})\end{array}$ & $\begin{array}{c}\boldsymbol{d = 1 - 3} \\
\text { días }\end{array}$ & $\begin{array}{c}\boldsymbol{d}=\mathbf{4 - 7} \\
\text { días }\end{array}$ & $\begin{array}{c}\boldsymbol{d = 8 - 1 1} \\
\text { días }\end{array}$ & $\boldsymbol{d}>\mathbf{1 1}$ días & $\begin{array}{c}\text { Daño total } \\
\text { promedio, } \\
\text { DTP }\end{array}$ \\
\hline 0.1 & 931.55 & 15424.99 & 24027.63 & 243854.84 & $\mathbf{2}$ \\
\hline
\end{tabular}

El procedimiento descrito anteriormente se desarrolla para cada escenario analizado asociado; para este estudio, a distintos periodos de retorno e intervalos propuestos de duración de inundación. La Tabla 6 muestra el concentrado de los resultados obtenidos, al igual que el daño total promedio $(D T P)$.

Tabla 6. Resumen del daño por escenario e intervalo de duración de la inundación en cultivo de maíz.

\begin{tabular}{|c|c|c|c|c|c|}
\hline \multirow{2}{*}{$\begin{array}{l}\text { Probabilidad } \\
\text { de excedencia } \\
\qquad P=1 / T r\end{array}$} & \multicolumn{4}{|c|}{ Daño (\$) } & \multirow{2}{*}{$\begin{array}{l}\text { Daño total } \\
\text { promedio } \\
\text { DTP (\$) }\end{array}$} \\
\hline & $d=1-3$ días & $\begin{array}{c}d=4-7 \\
\text { días }\end{array}$ & $\begin{array}{c}d=8-11 \\
\text { días }\end{array}$ & $d>11$ días & \\
\hline 0.5 & 269.40 & 9623.88 & 14073.76 & 42357.71 & 66324.74 \\
\hline 0.2 & 467.88 & 9833.28 & 23336.54 & 146470.66 & 180108.36 \\
\hline 0.1 & 931.55 & 15424.99 & 24027.63 & 243854.84 & 284239.01 \\
\hline 0.05 & 1182.50 & 17965.47 & 31998.37 & 319278.51 & 370424.85 \\
\hline 0.02 & 1667.63 & 44088.07 & 80416.56 & 341513.53 & 467685.80 \\
\hline 0.01 & 3402.36 & 81009.58 & 155324.33 & 516301.68 & 756037.96 \\
\hline 0.005 & 20954.31 & 103346.37 & 228141.93 & 532651.19 & 885093.81 \\
\hline 0.002 & 38837.69 & 237911.50 & 301377.37 & 638282.05 & 1216408.62 \\
\hline
\end{tabular}

En la Figura 14 se muestran las curvas para obtener el riesgo como el daño anual esperado en el cultivo de maíz, diferenciadas por intervalo de duración de la inundación. De igual forma se construyó la curva de riesgo para el daño total promedio para cada $T r$, con coordenadas (DTP, Tr). 
Utilizando la ecuación (3), el área bajo la curva de riesgo da un valor del daño anual esperado promedio (DAEP) de \$102 494.29 por hectárea.

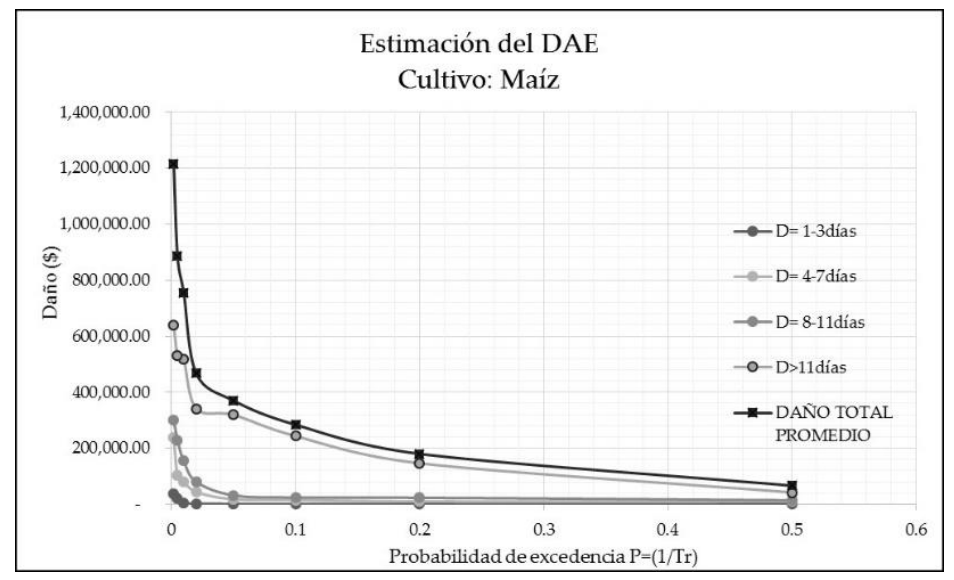

Figura 14. Daño anual esperado ( $D A E)$ para el cultivo de maíz.

\section{Conclusiones}

En el presente trabajo se generó una metodología aplicable a zonas con disponibilidad limitada de información de inundaciones en cultivos, sustentada en simulaciones hidrodinámicas bidimensionales. La metodología propuesta permite estimar el daño anual esperado, considerando duración, estacionalidad y profundidad de la inundación para construir las funciones de daño. Debido a que no se cuenta con una base de datos de daños en el sector agrícola que considere dichos parámetros para generar las curvas de daños, se tomaron como referencia criterios citados en la literatura, así como entrevistas con agricultores, investigadores y funcionarios del sector agropecuario. La construcción de las funciones de daño considera una combinación de la etapa de desarrollo del cultivo con cuatro rangos de duración de la inundación, con el objetivo de cuantificar desde una disminución en la producción hasta la pérdida total del cultivo. Por otro lado, con respecto al parámetro de la profundidad del agua, en el análisis se consideró que el daño ocurre a partir de que la celda está mojada y hasta el tirante 
máximo, debido a que el daño en el cultivo por inundación inicia por la disminución del oxígeno en el suelo, que genera un deterioro en la raíz de la planta y, como consecuencia, un inadecuado desarrollo y rendimiento.

El modelo propuesto para la evaluación de las pérdidas en los cultivos se aplicó a la cuenca del río Champotón, que por su ubicación se ha visto expuesta a diversas inundaciones históricas asociadas con la presencia de huracanes; entre los registrados con mayor severidad destacan Gilberto (1988), Opal y Roxanne (1995), e Isidore (2002). Los resultados de este trabajo son parte importante para la evaluación del riesgo por inundaciones asociadas con el río Champotón, y pueden ser utilizados por los tomadores de decisiones de las diferentes entidades de gobierno para establecer de manera más acertada los planes de ordenamiento, así como, por otro lado, para ajustar las primas de los seguros agropecuarios.

Es importante mencionar que la disponibilidad de información del uso de suelo es limitada, pues no contiene una tipología detallada de los cultivos en la zona, propiciando simplificaciones en la metodología. Sin embargo, se recomienda realizar un análisis más detallado sobre los cultivos que se desarrollan en la zona, como el sorgo, arroz palay, soya, pastizal y caña de azúcar, considerando patrones de cultivo más complejos, que incluyan la rotación de cultivos entre los ciclos primavera-verano (P-V) y otoñoinvierno (O-I). Asimismo, es necesario incluir en la estimación de daños otros elementos que intervienen en la agricultura, como maquinaria agrícola, edificios y silos, entre otros. Lo anterior implica generar información en campo más detallada a nivel de parcela.

Finalmente, se recomienda mejorar las bases de datos históricas sobre daños en el área agrícola, lo que permitirá validar los resultados obtenidos. De igual manera, por ser una parte fundamental en la estimación de los daños, las funciones o curvas de daño propuestas deben actualizarse en la medida de lo posible con bases de datos que se estén generando y con datos experimentales.

\section{Agradecimientos}

Al Programa de Mejoramiento del Profesorado (Promep), por el apoyo económico otorgado a Beatriz Edith Vega Serratos para la realización de sus estudios de doctorado. A la M. C. Débora Libertad Ramírez Vargas por su apoyo con las modelaciones hidráulicas. Al personal académico del INIFAP, campo experimental Edzna-Campeche, por las entrevistas 
concedidas relacionadas con diversos tipos de cultivos. Los autores agradecen a los colegas académicos por las revisiones hechas a este trabajo.

\section{Referencias}

Agraz-Hernández, C. M., Chan-Keb, C. A., Iriarte-Vivar, S. I., PosadaVanegas, G., Vega-Serratos, B. E., \& Osti-Sáenz, J. (2015). Phenological variation of Rhizophora mangle and groundwater chemistry associated to changes of the precipitation. Hidrobiológica, 25(1), 49-61.

Brémond, P., Grelot, F., \& Agenais, A. L. (2013). Review article: Economic evaluation of flood damage to agriculture- review and analysis of existing methods. Nat. Hazards Earth Syst. Sci., 13, 2493-2512. Recuperado de www.nat-hazards-earth-syst-sci.net/13/2493/2013/

Brémond, P., \& Grelot, F. (2010). Comparison of a systemic modelling of farm vulnerability and classical methods to appraise flood damage on agricultural activities. In: Advancing Sustainability in a Time of Crisis. 11th biennial conference of the International Society for Ecological Economics, August 22-25, Oldenburg and Bremen, Germany. Recuperado de https://hal.archives-ouvertes.fr/hal-00615462/document

Brémond, P., \& Grelot, F. (2012). Taking into account recovery to assess vulnerability: application to farms exposed to flooding. In: R. Seppelt, A. A. Voinov, S. Lange, D. Bankamp, \& International Environmental Modelling and Software Society (eds.) (Managing Resources of a Limited Planet). 2012 International Congress on Environmental Modelling and Software, Leipzig, Germany. Recuperado de http://www.iemss.org/sites/iemss2012//proceedings/H3_0429_Bremon da_\%20Grelot.pdf

Citeau, J. M. (April, 2003). A new flood control concept in the Oise catchment area: Definition and assessment of flood compatible agricultural activities. FIG Working Week, Paris, France (pp. 13-17). Recuperado https://www.fig.net/resources/proceedings/fig_proceedings/fig_2003/TS _14/TS14_5_Citeau.pdf

Chatterton, J., Viavattene, C., Morris, J., Penning-Rowsell, E., \& Tapsell, S. (2010). The costs of the Summer 2007 Floods in England (Technical report). Environment Agency. Recuperado de http://nationalfloodforum.org.uk/wp-content/uploads/EA-Costs-ofFlooding.pdf 
Chau, V. N., Cassells, S., \& Holland, J. (September 5, 2014). Economic impact upon agricultural production from extreme flood events in Quang Nam, central Vietnam. Nat Hazards. DOI: 10.1007/s11069-014-1395-x

DHI, Water \& Environment. (2014a). Mike 21 y Mike 3 Flow Model FM. Hydrodynamic and Transport Module. Scientific Documentation (10 ed.). Hørsholm, Denmark: DHI, Water \& Environment.

DHI, Water \& Environment. (2014b). Mike 21 y Mike 3 Flow Model FM. Hydrodynamic Module. Step-by-step training guide. Hørsholm, Denmark: DHI, Water \& Environment.

Domínguez, R., Esquivel, G., Méndez, B., Mendoza, A., Arganis, M. L., \& Carrizosa, E. (2008). Manual del modelo para pronóstico de escurrimiento. Series. México, DF, México: Instituto de Ingeniería de la Universidad Nacional Autónoma de México.

Dutta, D., Herath, S., \& Musiake, K. (2003). A mathematical model for flood loss estimation. Journal of Hydrology, 277(1), 24-49. DOI: $10.1016 / \mathrm{s} 0022-1694(03) 00084-2$

Escalante-Sandoval, C. A., \& Reyes-Chávez, L. (2002). Técnicas estadísticas en hidrología. México, DF, México: Universidad Nacional Autónoma de México, Facultad de Ingeniería.

Förster, S., Kuhlmann, B., Lindenschmidt, K. E., \& Bronstert, A. (2008). Assessing flood risk for a rural detention area. Natural Hazards and Earth System Sciences, 8, 311-322. DOI: 10.5194/nhess-8-311-2008

Guevara, O., Quaas, W., \& Fernández, V. (2006). Lineamientos generales para la elaboración de atlas de riesgos. En: Conceptos básicos sobre peligros, riesgos y representación geográfica. Serie: Atlas Nacional de Riesgos. México, DF, México: Secretaría de Gobernación.

Jiménez, E. M. (1992). AX (Ajuste de Función de Probabilidad) versión 1.05. México, DF, México: Centro Nacional de Prevención de Desastres, Facultad de Ingeniería de la Universidad Nacional Autónoma de México.

Kadam, P., \& Sen, D. (2012). Flood inundation simulation in Ajoy River using Mike Flood. ISH Journal of Hydraulic Engineering, 18(2), 129-141.

Li, K., Wu, S., Dai, E., \& Xu, Z. (September, 2012). Flood loss analysis and quantitative risk assessment in China. Natural Hazards, 63(2), 737760. DOI: $10.1007 / \mathrm{s} 11069-012-0180-y$ 
Martínez, A. (2011). Estudio hidrológico e hidráulico de la cuenca del río Champotón: mapas de peligro y sistemas de información geográfica (tesis de licenciatura). Campeche, México: Universidad Autónoma de Campeche, Facultad de Ciencias Químico-Biológicas.

Merz, B., Kreibich, H., Schwarze, R., \& Thieken, A. (2010). Review article "Assessment of economic flood damage". Natural Hazards and Earth System Sciences, 10, 1697-1724. DOI:10.5194/nhess-10-1697-2010

Meyer, V., \& Messner, F. (2005). National Flood Damage Evaluation Methods. A Review of Applied Methods in England, The Netherlands, The Czech Republic and Germany (FLOODsite Project Report Contract No:GOCE-CT-2004-505420). Leipzig, Germany: The Helmholtz-Centre for Environmental Research GmbH-UFZ, Department of Economics.

Meyer, V., Scheuer, S., \& Haase, D. (2009). A multicriteria approach for flood risk mapping exemplified at the Mulde River, Germany. Natural Hazards, 48,17-39.

Meyer, V., Priest, S. \& Kuhlicke, C. (2012). Economic evaluation of structural and non-structural flood risk management measures: Examples from the Mulde River. Natural Hazards, 62(2), 301-324. DOI: $10.1007 / \mathrm{s} 11069-011-9997-z$

Morris, J., \& Brewin, P. (2013). The impact of seasonal flooding on agriculture: The spring 2012 floods in Somerset, England. Flood Risk Management, DOI: $10.1111 / \mathrm{jfr} 3.12041$

Patro, S., Chatterjee, C., Mohanty, S., Singh, R., \& Raghuwanshi, N. S. (2009). Flood inundation modelling using MIKE FLOOD and remote sensing data. Journal of the Indian Society of Remote Sensing, 37(1), 107-118. DOI: $10.1007 / \mathrm{s} 12524-009-0002-1$

Pistrika, A. (2010). Flood damage estimation based on flood simulation scenarios and a GIS platform. European Water, 30, 3-11.

Pivot, J.-M., Josien, E., \& Martin, P. (2002). Farms adaptation to changes in flood risk: A management approach. Journal of Hydrology, 267(1-2), 12-25.

Posada, G., Veja, B., Ruiz, G., Echávez-Aldape, G., \& Martínez, A. (2011). Áreas inundables para la cuenca del río Champotón, Campeche: escenarios actuales y futuros. En: A. V. Botello, S. Villanueva-Fragoso, J. Gutiérrez, \& J. L. Rojas-Galaviz (eds.). Vulnerabilidad de las zonas costeras mexicanas ante el cambio climático (2a ed.). México, DF, México: Secretaría de Medio Ambiente y Recursos Naturales-Instituto Nacional de 
Ecología y Cambio Climático, Universidad Nacional Autónoma de MéxicoInstituto de Ciencias del Mar y Limnología, Universidad Autónoma de Campeche.

Posada-Vanegas, G., Vega-Serratos, B. E., \& Silva-Casarín, R. (eds.). (2013). Peligros naturales en el estado de Campeche. Cuantificación y Protección Civil. Campeche, México: Universidad Autónoma de Campeche, Centro Estatal de Emergencias de Campeche-Gobierno del Estado de Campeche, Centro Nacional de Prevención de Desastres.

Posthumus, H., Morris, J., Hess, T. M., Neville, D., Philips, E., \& Baylis, A. (2009). Impacts of the summer 2007 floods on agriculture in England. Journal of Flood Risk Management, 2, 182-189.

Ramírez, V. D. L. (2015). Modelación de escenarios en las zonas inundables ante la construcción de un desvío carretero en la ciudad de Champotón, Campeche (Tesis de Maestría). Campeche, México: Universidad Autónoma de Campeche, Facultad de Ciencias QuímicoBiológicas.

Ritchie, S., \& Hanway, J. (1982). How a corn plant develops (Special Report No 48). Ames, USA: Iowa State University, Cooperative Extension Service.

Roca, M., Bast, H., Panzeri, M., Hess, T. M., Sayers, P., Flikweert, J., Ogunyoye, F., \& Young, R. (2011). Developing the evidence base to describe the flood risk to agricultural land in England and Wales, R\&D (Technical Report FD2634/TR). London, UK: Department for Environment, Food and Rural Affairs.

Secretaría de Agricultura, Ganadería, Desarrollo Rural, Pesca y Alimentación, Sagarpa. (2009). PRONAC. "Digitalización del Campo Cañero en México para alcanzar la Agricultura de Precisión de la Caña de Azúcar". Desarrollo de un Modelo Integral de SIG y Edáfica como Fundamento de la Agricultura de Precisión en la Caña de Azúcar en México. Etapa I. San Francisco de Campeche, México: Secretaría de Agricultura, Ganadería, Desarrollo Rural, Pesca y Alimentación.

Secretaría de Agricultura, Ganadería, Desarrollo Rural, Pesca y Alimentación, Sagarpa. (2015). Agenda técnica agrícola. Campeche. Campeche, México: Secretaría de Agricultura, Ganadería, Desarrollo Rural, Pesca y Alimentación.

Servicio de Información Agroalimentaria y Pesquera-Secretaría de Agricultura, Ganadería, Desarrollo Rural, Pesca y Alimentación, SIAPSagarpa (2014). SIAP. Recuperado de 
http://www.sagarpa.gob.mx/quienesomos/datosabiertos/siap/Paginas/d efault.aspx

Smith, D. I. (1994). Flood damage estimation-a review of urban stagedamage curves and loss functions. Water SA, 20(3), 231-238.

Sommer, T., Karpf, C., Ettrich, N., Haase, D., Weichel, T., Peetz, J.-V., Steckel, B., Eulitz, K., \& Ullrich, K. (2009). Coupled modelling of subsurface water flux for an integrated flood risk management. Natural Hazards and Earth System Sciences, 9, 1277-1290. DOI:10.5194/nhess9-1277-2009

Thieken, A. H., Ackermann, V., Elmer, F., Kreibich, H., Kuhlmann, B., Kunert, U., Maiwald, H., Merz, B., Müller, M., Piroth, K., Schwarz, J., Schwarze, R., Seifert, I., \& Seifert, J. (May 6-8, 2009). Methods for the evaluation of direct and indirect flood losses. In: Proceedings. 4th International Symposium on Flood Defense: Managing Flood Risk, Reliability and Vulnerability, Toronto, Ontario, Canada.

Twining, S., Procter, C., Wilson, L., Frost, A., Phillips, K., Turner, T., Tiffin, D., Emery, J., Walker, O., Welch, S., \& Wells, A. (2007). Impacts of 2007 Summer Floods on Agriculture, Tech. Rep. Final. Cambridge, UK: ADAS.

US Army Corps of Engineers, USACE. (1985). AGDAM, Agricultural Flood Damage Analysis, User's Manual (Provisional), Computer Program Documentation CPD-48. Davis, USA: US Army Corps of Engineers, Institute for Water Resources, Hydrologic Engineering Center (HEC).

Van Westen, C. J. (2010). GIS for the assessment of risk form geomorphological hazards. In: I. Alcántara-Ayala \& A. S. Goudie (eds.). Geomorphological Hazards and Disasters Preventions (pp. 205-219). Cambridge, UK: Cambridge University Press.

Vozinaki, A. E., Karatzas, G., Sibetheros, I., \& Varouchakis, E. (2015). An agricultural flash flood loss estimation methodology: The case study of the Koiliaris basin (Greece), February 2003 flood. Natural Hazards, 79, 899-920. DOI: $10.1007 /$ s11069-015-1882-8

Yu, J. J., Qin, X. S., \& Larsen, O. (2013). Joint Monte Carlo and possibilistic simulation for flood damage assessment. Stochastic Environmental Research and Risk Assessment, 27(3), 725-735. DOI: 10.1007/s00477012-0635-4 